\title{
A Role of Actin Filament in Synaptic Transmission and Long-Term Potentiation
}

\author{
Chong-Hyun Kim and John E. Lisman \\ Department of Neuroscience and Volen Center for Complex Systems, Brandeis University, \\ Waltham, Massachusetts 02454
}

\begin{abstract}
The role of actin filaments in synaptic function has been studied in the CA1 region of the rat hippocampal slice. Bath application (2 hr) of the actin polymerization inhibitor latrunculin B did not substantially affect the shape of dendrites or spines. However, this and other drugs that affect actin did affect synaptic function. Bathapplied latrunculin B reduced the synaptic response. Several lines of evidence indicate that a component of this effect is presynaptic. To specifically test for a postsynaptic role for actin, latrunculin B or phalloidin, an actin filament stabilizer, was perfused into the postsynaptic neuron. The magnitude of long-term potentiation
\end{abstract}

(LTP) was decreased at times when baseline transmission was not yet affected. Longer applications produced a decrease in baseline AMPA receptor (AMPAR)-mediated transmission. The magnitude of the NMDA receptor-mediated transmission was unaffected, indicating a specific effect on the AMPAR. These results suggest that postsynaptic actin filaments are involved in a dynamic process required to maintain AMPAR-mediated transmission and to enhance it during LTP.

Key words: actin filament; latrunculin $B$; cytochalasin $D$; phalloidin; LTP; CA1; hippocampus; AMPAR; NMDAR
Actin is one of the major cytoskeletal proteins. In neurons, the existence of actin filaments in both the presynaptic terminals and postsynaptic spines has been well documented (Kelly and Cotman, 1978; Fifkova and Delay, 1982; Matus et al., 1982; Cumming and Burgoyne, 1983; Drenckhahn and Kaiser, 1983; Cohen et al., 1985; Landis, 1988; Kaech et al., 1997; Fisher et al., 1998). In the presynaptic terminal, actin filaments interact with synaptic vesicles in a process that involves synapsins (Greengard et al., 1993; Sudhof, 1995; Calakos and Scheller, 1996). Postsynaptic dendritic spines contain a much higher concentration of actin than dendrites (Matus et al., 1982; Cohen et al., 1985; Fifkova, 1985). Actin filaments directly contact the postsynaptic density (PSD) and vesicular structures (Gulley and Reese, 1981; Fifkova and Delay, 1982; Matus et al., 1982; Markham and Fifkova, 1986).

Several studies suggest that there may be a functional role of postsynaptic actin filaments in the synaptic function. Stabilization of actin filament blocks the use-dependent rundown of NMDA receptor (NMDAR) current evoked by extracellular NMDA application (Rosenmund and Westbrook, 1993). $\alpha$-Actinin 2, an actin binding protein, interacts directly with the NR1A/1C/2B subunits of NMDAR (Wyszynski et al., 1997; Allison et al., 1998). The mechanosensitivity of the NMDAR also indirectly suggests the interaction of NMDAR with cytoskeletal structures (Paoletti and Ascher, 1994). Actin also plays an important role in the clustering of AMPA receptor (AMPAR) and NMDAR channels (Allison et al., 1998). Finally, recent work demonstrates that

Received Dec. 28, 1998; revised March 17, 1999; accepted March 22, 1999.

This work was supported by the National Institutes of Health Grant 5 RO1 NS27337-09. We gratefully acknowledge the support of the W. M. Keck Foundation. We gratefully thank Dr. Nikolai Otmakhov for providing software and help for whole-cell experiment and helpful discussions throughout the experiment. We also thank Drs. Sacha Nelson and Leslie Griffith for their careful reading of this manuscript. We thank Dr. Ole Jensen, Dr. Nonna A. Otmakhova, Dr. Ed Richard, Natalia Slutskaya, and Lindsay Mortenson for their support.

Correspondence should be addressed to John E. Lisman, Volen Center for Complex Systems, Brandeis University, Waltham, MA 02254.

Copyright (C) 1999 Society for Neuroscience $0270-6474 / 99 / 194314-11 \$ 05.00 / 0$ spines undergo continuous submicrometer movements that are dependent on actin (Fisher et al., 1998).

There have also been suggestions that actin filaments may play a role in long-term potentiation (LTP). An increase in actin filament bundles was observed after tetanus-induced LTP in the diffuse cytoskeletal meshwork that connects the dendritic cytoplasm to the spine matrix (Pavlik and Moshkov, 1992). There are indications that LTP produces a segmentation of PSDs into independent regions (Geinisman et al., 1991), and it has been suggested that this and other morphological changes in spines may be actin-dependent (Fifkova and Morales, 1992; Edwards, 1995). Recently, it has been shown that LTP requires a postsynaptic membrane fusion process (Lledo et al., 1998), a process that might depend on actin.

These results suggest that actin may play a dynamic role in synaptic function, but the physiological role of actin in synaptic transmission and LTP has not been previously investigated. We have examined the effects of the actin polymerization inhibitors (APIs) latrunculin B and cytochalasin D (Spector et al., 1983, 1989) and the actin filament stabilizer phalloidin (Cooper, 1987) on synaptic physiology in the CA1 region of the rat hippocampal slice. Our results indicate a requirement for presynaptic and postsynaptic actin function in basal synaptic transmission and synaptic plasticity.

\section{MATERIALS AND METHODS}

Hippocampal slices $(400 \mu \mathrm{m})$ were prepared from 2- to 3-week-old Long-Evans rats as described previously (Otmakhov et al., 1997). In brief, slices were allowed to recover for a minimum of $2 \mathrm{hr}$ on the surface of cell culture inserts in an incubation chamber to which humidified oxygen was continuously supplied $\left(95 \% \mathrm{O} 2-5 \% \mathrm{CO}_{2}\right)$ and then transferred to a submerged type recording chamber with continuous flow (2.3 $\mathrm{ml} / \mathrm{min}$ ) of oxygenated artificial CSF (ACSF) at $35^{\circ} \mathrm{C}$. The ACSF for recording contained (in $\mathrm{mM}$ ): $\mathrm{NaCl} 124, \mathrm{NaHCO} 326, \mathrm{NaH}_{2} \mathrm{PO}_{4} 1.25$, $\mathrm{KCl} 2.5, \mathrm{CaCl} 24, \mathrm{MgSO}_{4} 4$, D-glucose 20, and picrotoxin 0.05, $\mathrm{pH} 7.3$. Whole-cell recording pipette was filled with (in $\mathrm{mm}$ ): Csmethanesulfonate 130, CsCl 20, HEPES 10, MgATP 1, Na3GTP 0.4, EGTA 0.2 , and phosphocreatine 15, pH 7.3 (with osmolarity at $300 \mathrm{mOsm}$ ). In the phalloidin experiment, $2 \mathrm{~mm}$ MgATP was used. In NMDAR- 


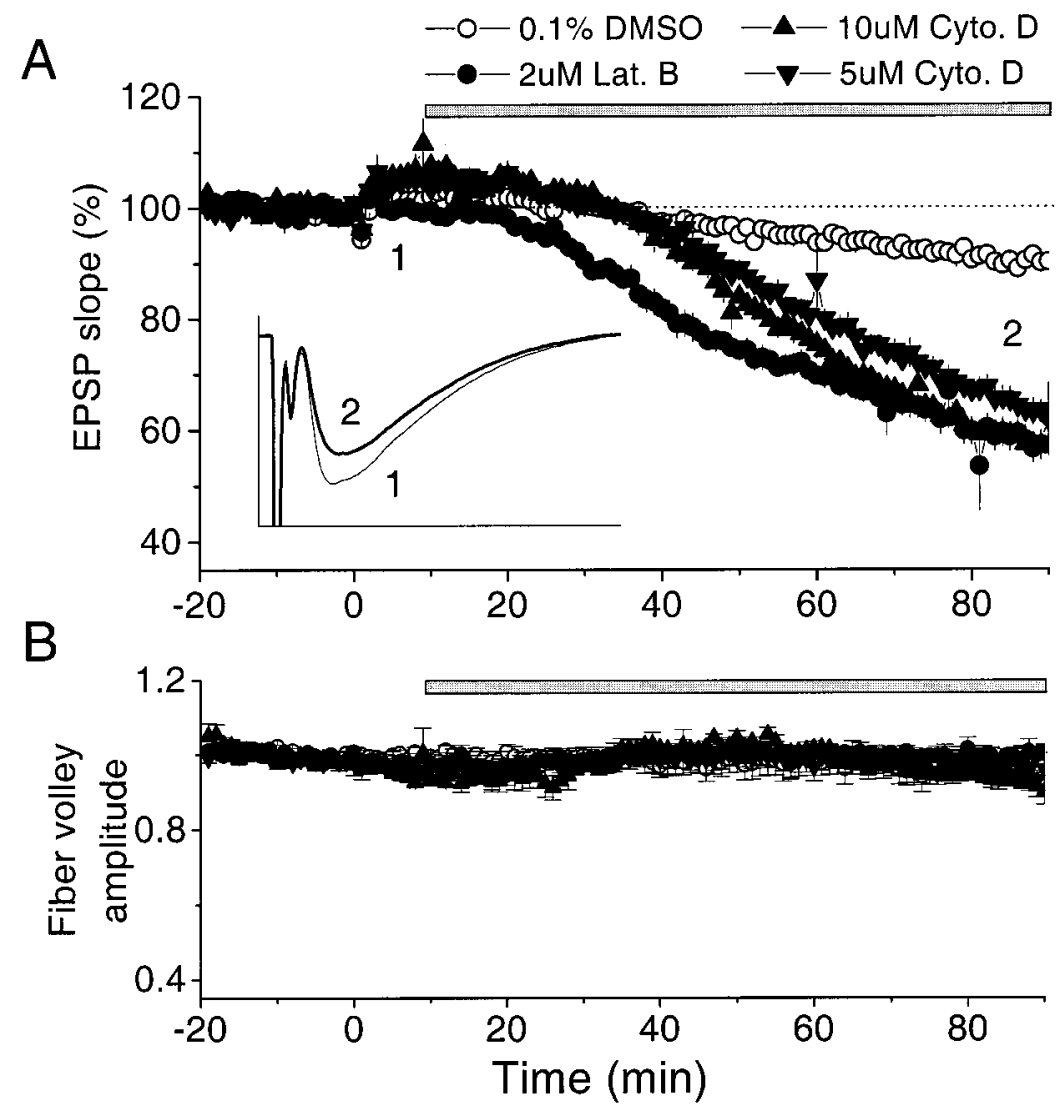

Figure 1. Bath-applied latrunculin B and cytochalasin D decrease fEPSP in CA1 region of hippocampal slice. $A$, Actin polymerization inhibitors latrunculin $\mathrm{B}(2 \mu \mathrm{M} ; n=7)$ or cytochalasin D (5 $\mu \mathrm{M} ; n=7 ; 10 \mu \mathrm{M} ; n=4)$ were applied extracellularly dissolved in $0.1 \%$ DMSO. Control solution contained $0.1 \%$ DMSO $(n=10)$. The bar indicates the period of drug application. The average fEPSP in the DMSO controls, $2 \mu \mathrm{M}$ latrunculin $\mathrm{B}$, and $5 \mu \mathrm{M}$ and $10 \mu \mathrm{M}$ cytochalasin D experiments, measured at 70-80 min after its application, were $91,59,63$, and $57 \%$ of the baseline, respectively. The small rise at time 0 occurred because LTP was induced in a different pathway. Inset, Examples of average traces before (thin line, 1) and after $2 \mu \mathrm{M}$ latrunculin B application (thick line, 2). Calibration: $2.5 \mathrm{mV}, 25 \mathrm{msec}$. B, Effect of bath-applied APIs on fiber volley amplitude. The same symbols were used as in $A$. The effect on the amplitude of fiber volley of $0.1 \%$ DMSO ACSF $(n=7), 2 \mu \mathrm{M}$ latrunculin B $(n=7)$, and cytochalasin $\mathrm{D}(5 \mu \mathrm{M} ; n=7 ; 10 \mu \mathrm{M} ; n=4)$ were indistinguishable, indicating no effect of APIs on presynaptic excitability. mediated field EPSP (fEPSP) measurement, $0.1 \mathrm{mM} \mathrm{Mg}^{2+}$ and $10 \mu \mathrm{M}$ CNQX were used in ACSF. In whole-cell NMDAR-mediated EPSC measurement, $2.5 \mathrm{~mm} \mathrm{Ca}^{2+}, 1.3 \mathrm{~mm} \mathrm{Mg}^{2+}$, and $10 \mu \mathrm{M} \mathrm{CNQX}$ were in ACSF.

In both field and whole-cell experiments, two synaptic pathways were stimulated alternately. One pathway served as the control path and the other as the test path. Stimulation of Schaffer-commissural afferents was performed using two glass electrodes filed with ACSF. The independence of two synaptic pathways was tested by a paired-pulse protocol. Paired-pulse facilitation (PPF) of EPSPs and EPSCs was observed only when two consecutive pulses with an interval $50 \mathrm{msec}$ were applied to the same path. When two consecutive pulses were applied to different pathways, no facilitation was observed. In field recording experiments, the stimulus interval was $1 \mathrm{~min}$. Traces were filtered at $1 \mathrm{kHz}$. Both the slope and the amplitude of the fEPSP were measured to quantify the magnitude of fEPSP responses. The time window for the slope measurement was $1 \mathrm{msec}$ starting $0.2 \mathrm{msec}$ after the time of minimum voltage between the fiber volley and the fEPSP. This window corresponded to $\sim 4-65 \%$ of the peak-to-peak amplitude of the fEPSP. The slope was calculated by a linear regression method. In the figures, slope measurements are shown, but analysis based on peak amplitude gave the same result. Fiber volley amplitude was measured to monitor axon excitability. Fiber volley was measured as a separation between the peak of fiber volley and the line connecting the beginning and end of the fiber volley. LTP was induced by a $100 \mathrm{~Hz}$ theta-burst protocol: $100 \mathrm{~Hz}$, five pulses per burst; 10 bursts at $200 \mathrm{msec}$ intervals. A $25 \mathrm{~Hz}$ theta-burst protocol was used for some experiments: $25 \mathrm{~Hz}$, five pulses per burst; 10 bursts at $120 \mathrm{msec}$ interval. During induction, no stimuli were delivered to the control path. After the theta burst, there was a 2 min delay before the first fEPSP response.

In whole-cell experiments, cells were held at $-65 \mathrm{mV}$ using an Axopatch 1D (Axon Instruments, Foster City, CA) amplifier. Stimulus interval was $6 \mathrm{sec}$. Series resistance (5-12 $\mathrm{M} \Omega$ ) and input resistance ( $70-200 \mathrm{M} \Omega$ ) were monitored every $6 \mathrm{sec}$ by measuring the peak and steady-state currents in response to $2 \mathrm{mV}, 30 \mathrm{msec}$ hyperpolarizing steps. Holding current was also monitored throughout the experiment. For monitoring the stability of the slice responsiveness, the amplitude of fEPSP was recorded simultaneously while measuring the amplitude of EPSC. Data were filtered at $1 \mathrm{kHz}$. Whole-cell LTP was induced by pairing: $2 \mathrm{~Hz}, 200$ pulses during depolarization to $0 \mathrm{mV}$. Changing of the internal pipette solution was done as described previously (Otmakhov et al., 1997). Experiments with $\geq 13 \mathrm{M} \Omega$ series resistance were discarded. Responses were averaged at 1 (field) or 2 (whole-cell) min intervals and then normalized to the average of baseline recording before either LTP induction or drug application. The magnitude of LTP was measured as a percentage of LTP path response over that of non-LTP path response at a given time. In miniature EPSC (mEPSC) experiments, $8 \mathrm{~mm} \mathrm{Sr}^{2+}$ was used instead of $4 \mathrm{~mm} \mathrm{Ca}^{2+}$ in ACSF, and data were acquired as described by Oliet et al. (1996). In brief, after the EPSC amplitude reached a steady level in the presence of $\mathrm{Sr}^{2+}$, each pathway was stimulated alternatively five times (every $30 \mathrm{sec}$ ) at $2 \mathrm{~Hz}$ for $10 \mathrm{sec}$. mEPSCs were picked by considering their peak amplitude (approximately $>3.5 \mathrm{pA}$ ) and duration at half peak amplitude. mEPSC amplitude was measured as difference of average value $(4 \mathrm{msec})$ between the peak and the baseline before the mEPSC. After collecting control data, latrunculin $\mathrm{B}$ was applied for $30 \mathrm{~min}$ before data collection.

All data acquisition and analysis were done by custom software written in Axobasic 3.1 (Axon Instruments). Mean \pm SEM was used for representing average values. Error bars in graphs indicate SEM. When average data were plotted, measurements were normalized to the average of baseline responses unless stated otherwise. In mEPSC frequency analysis, single-factor ANOVA was used. In assessing the significance of effect on LTP of drugs and on mEPSC amplitude, a Kolmogorov-Smirnov (K-S) test was used as described previously (Cohen et al., 1992). Latrunculin $\mathrm{B}$, cytochalasin $\mathrm{D}$, and phalloidin were purchased from $\mathrm{Cal}-$ biochem (La Jolla, CA). The data were compiled in Microsoft (Seattle, WA) Excel and plotted using Microcal Origin (Microcal Software Inc., Northhampton, MA).

For confocal microscopy (MRC 600; Bio-Rad, Hercules, CA), the COMOS program (Bio-Rad) was used in acquiring pictures, and the Adobe Systems (San Jose, CA) Photoshop program was used to print pictures. DiI (Molecular Probes, Eugene, OR) was used to label the neuronal membrane. DiI was dissolved at saturation level in a commercial vegetable oil. A drop of DiI was applied onto the soma of CA1 pyramidal neurons through pipette. A syringe was used to generate the pressure for dropping DiI. Imaging was done on CA1 pyramidal cells in the slice to which ACSF was perfused continuously. Pictures were taken at $30 \mathrm{~min}$ intervals. 


\section{RESULTS}

\section{Bath-applied APIs reduce the fEPSP}

The effect of APIs on synaptic transmission was first studied by recording fEPSPs in the dendritic region of CA1 rat hippocampal slices (Fig. 1). These responses were quantified by measuring the slope of the early rising phase, which is almost exclusively a result of the AMPAR-mediated synaptic transmission (Collingridge et al., 1983; Wigstrom and Gustafsson, 1986). After $30 \mathrm{~min}$ of monitoring basal synaptic responses, $2 \mu \mathrm{M}$ latrunculin B (dissolved in $0.1 \%$ DMSO) was applied. It slowly reduced the fEPSP. After $80 \mathrm{~min}$, the fEPSP was reduced by $41 \%$ compared with the initial level $(n=7)$. As a control, ACSF containing $0.1 \%$ DMSO was applied; the fEPSP dropped by only $9 \%(n=10)$. This was comparable with the decline we observed without application of any drug. Compared with the effect of DMSO control, latrunculin $\mathrm{B}$ thus produced a reduction of the fEPSP of $35 \%$. Another API, cytochalasin D, produced similar effects on basal synaptic transmission after $80 \mathrm{~min}$ application. The reduction of the fEPSP compared with the DMSO control was $31 \%$ for $5 \mu \mathrm{M}(n=7)$ and $37 \%$ for $10 \mu \mathrm{M}(n=4)$ cytochalasin D. This effect of APIs began $\sim 10$ min after application and continued to increase throughout the application. We were able to obtain some slow reversal of the effect after washout of APIs, but this was never complete within $1 \mathrm{hr}$ (see Fig. 4A). The reduction of fEPSP by APIs was not caused by a drop in axonal excitability because the amplitude of the fiber volley, which was measured simultaneously, was not significantly affected $(n=7 ; t$ test; $p>0.05)$ (Fig. $1 B)$. To determine whether the effect of latrunculin $B$ requires synaptic stimulation, we turned off stimulation for 80 min during the application period (100 min). The results showed that the API effect still occurred under these conditions ( $n=3$; data not shown).

A possible explanation of the effect of APIs is that they produce a structural collapse of dendritic spines because actin filaments are important structural components of spines (Markham and Fifkova, 1986). To explore this possibility, pyramidal cells in the CA1 region of the hippocampal slice were labeled with DiI (see Materials and Methods). Spine and dendritic morphology was visualized in a confocal microscope before and after application of $4 \mu \mathrm{M}$ latrunculin B $(n=3)$. Two hours of application did not abolish postsynaptic spines or produce any obvious change in the shape of dendrites (Fig. $2 A, B$ ). Specifically, of 16 spines (on three dendrites in three different slices) identified before drug application, 15 were clearly visible after drug application and did not show any sign of structural collapse. This stability of spine shape is consistent with other published work. Allison et al. (1998) observed that the actin network within postsynaptic spines of cultured hippocampal neurons was not affected by $2 \mathrm{hr}$ incubation with $5 \mu \mathrm{M}$ latrunculin A or $24 \mathrm{hr}$ incubation with $20 \mu \mathrm{M}$ cytochalasin D. Similarly, Fisher et al. (1998) showed that ongoing submicrometer movements of actin filaments within spines are abolished by cytochalasin D or latrunculin B, but the overall structure of the actin network is unaffected.

\section{There is a presynaptic locus for the effect of bath-applied APIs on basal synaptic transmission}

Several lines of investigation were undertaken to determine whether bath-applied APIs worked presynaptically or postsynaptically. The measurements in Figure 1 indicate that APIs affected the AMPAR-mediated synaptic transmission. If bath-applied APIs affected transmitter release, the NMDAR-mediated synaptic transmission should also be affected. To test this possibility, the area of the NMDAR-mediated fEPSP was measured in 0.1
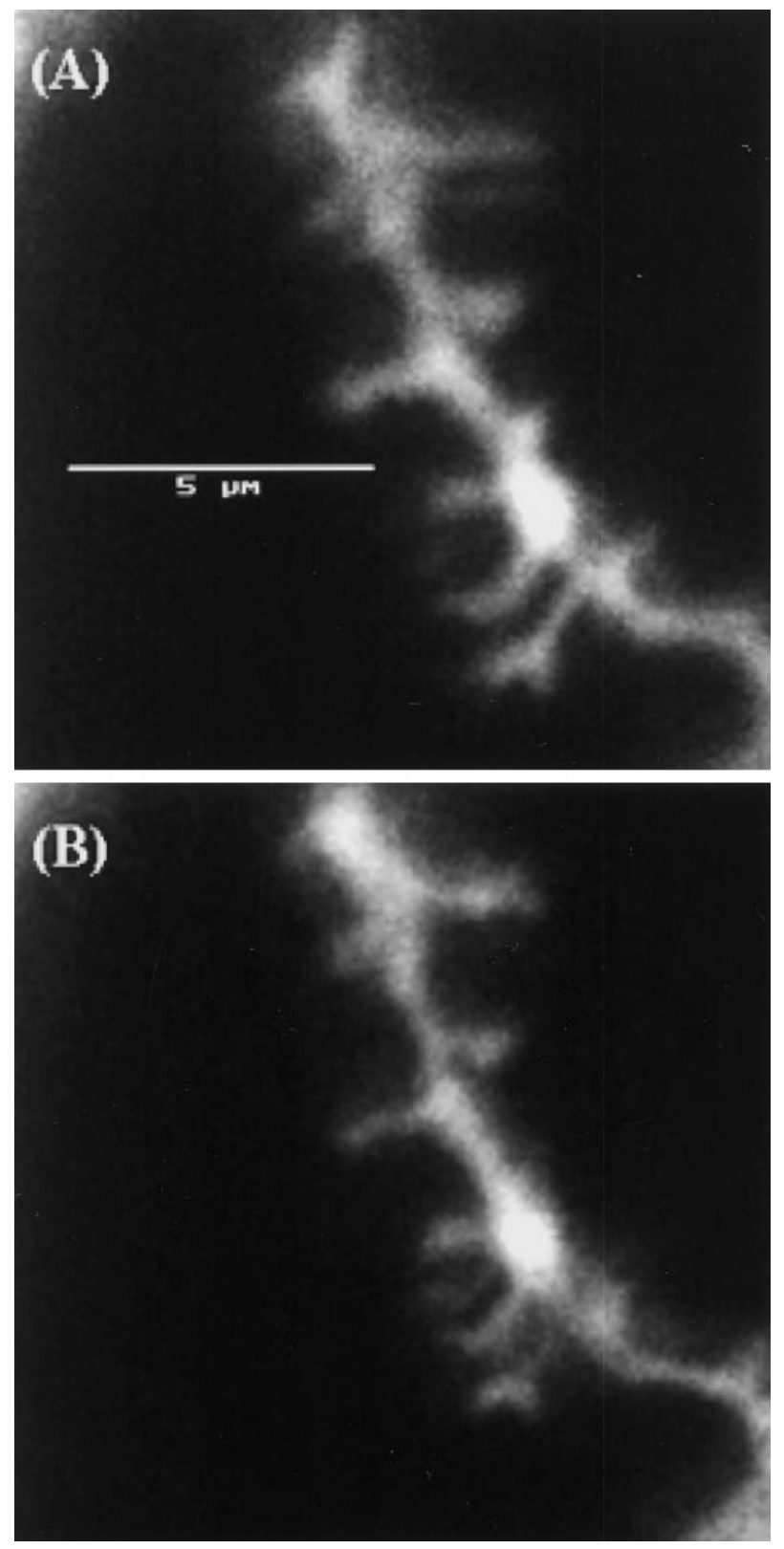

Figure 2. Bath-applied latrunculin B does not abolish postsynaptic spines or dendrites. Confocal image of dendrite of CA1 pyramidal neuron labeled with DiI. Before $(A)$ and after $(B) 2 \mathrm{hr}$ application of $4 \mu \mathrm{M}$ latrunculin B. Scale bar, $5 \mu \mathrm{m}$.

mM $\mathrm{Mg}^{2+}$ and $10 \mu \mathrm{M}$ CNQX, a blocker of AMPARs (Fig. $3 A$ ). Latrunculin B $(2 \mu \mathrm{M})$ decreased the NMDAR-mediated fEPSP by $40 \%$ in $80 \mathrm{~min}$. In interleaved DMSO control experiments, the NMDAR-mediated fEPSP dropped by $9 \%$. The reduction caused by latrunculin $\mathrm{B}$ was therefore $35 \%$. This figure is similar to that of the AMPAR-mediated fEPSP (35\%; see above). These results would be most simply explained by a reduction in transmitter release but do not rule out a postsynaptic site of action.

A second approach was to measure PPF, a classical method for locating the site of drug action (Creager et al., 1980; Charlton et al., 1982; Hess et al., 1987). The PPF ratio of two responses at 50 msec interval was measured. Latrunculin B $(2 \mu \mathrm{M})$ significantly increased the average PPF measured 56-65 min after application $(n=7 ; t$ test; $p \ll 0.01)$ (Fig. $3 B)$. This effect was not seen in the 


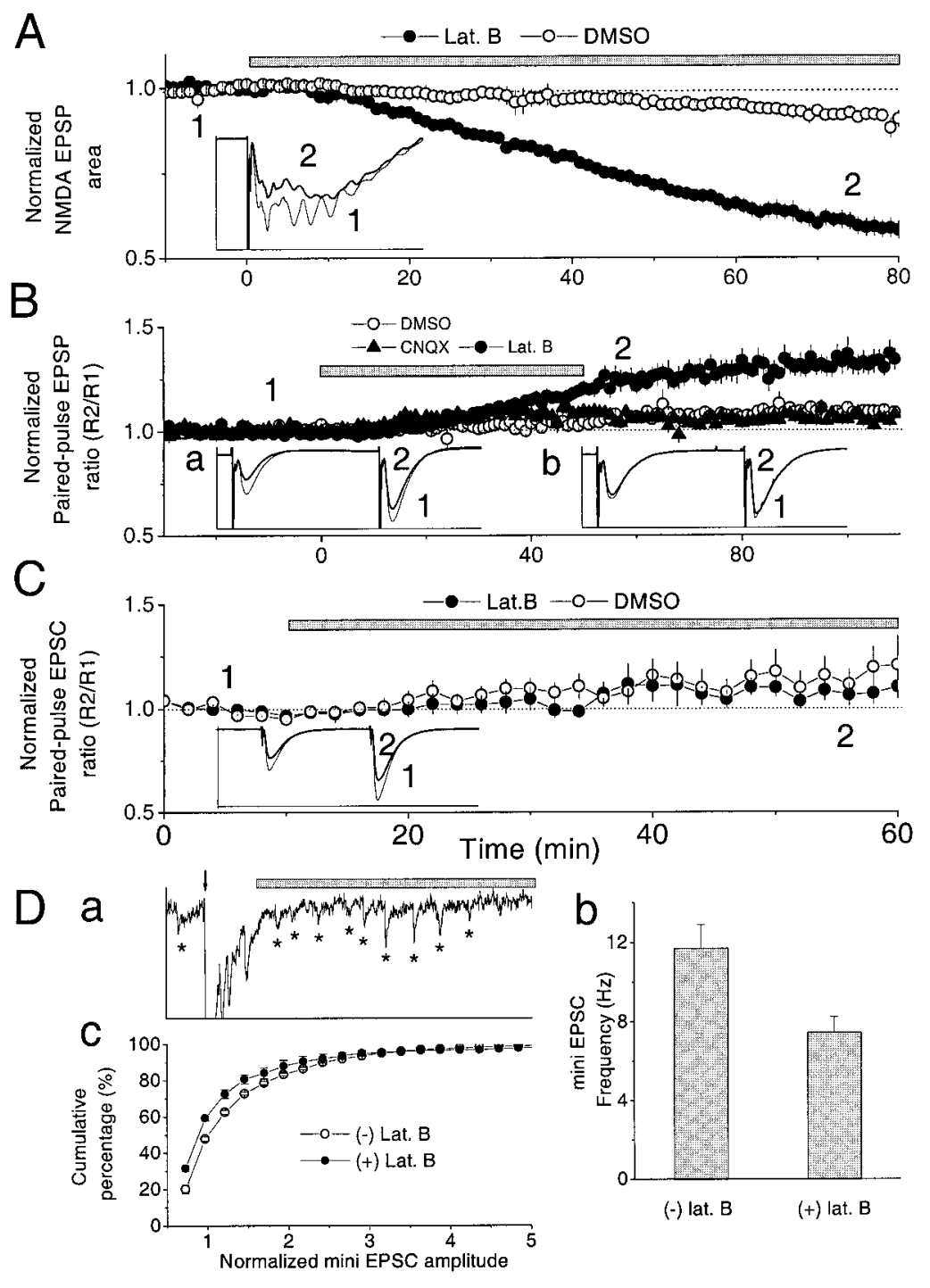

Figure 3. Experiments indicating that there is a presynaptic site of action of bath-applied latrunculin B. $A$, The NMDARmediated synaptic transmission was also reduced by latrunculin $B$. The area of the NMDAR-mediated fEPSP was measured with ACSF containing $0.1 \mathrm{~mm} \mathrm{Mg}^{2+}$ in which $10 \mu \mathrm{M}$ CNQX was used to block AMPAR-mediated synaptic transmission. The bar indicates the period of drug application. Latrunculin B $(2 \mu \mathrm{M})$ was applied $10 \mathrm{~min}$ after baseline recording $(n=6)$. As a control, $0.1 \%$ DMSO ACSF was used $(n=3)$. Inset, Average fEPSP before (thin trace, 1) and after (thick trace, 2) latrunculin $\mathrm{B}$ application. The spiking always appeared at this stimulation condition $\left(0.1 \mathrm{mM} \mathrm{Mg}^{2+}, 2.5 \mathrm{mM} \mathrm{Ca}^{2+}, 2.5 \mathrm{~mm} \mathrm{KCl}\right.$, and $50 \mu \mathrm{M}$ picrotoxin in ACSF, and without CA3 region in slice) and was blocked by APV (data not shown; Bortolotto and Collingridge, 1998). Calibration: $2 \mathrm{mV}, 100 \mathrm{msec}$. $B$, Paired-pulse facilitation quantified by the ratio of the initial slopes of two fEPSPs at 50 msec interval. The bar indicates the duration of drug application. Latrunculin B $(2 \mu \mathrm{M})$ was applied for $50 \mathrm{~min}$ after $20 \mathrm{~min}$ of baseline recording $(n=7)$. The average PPF ratios at 5-10 min after washout of DMSO control, 0.3 $\mu \mathrm{M}$ CNQX, and latrunculin B experiments were $1.07 \pm 0.009,1.06 \pm 0.02$, and $1.25 \pm 0.03$ (mean $\pm \mathrm{SE}$ ), respectively. Inset $a$, Examples of average responses before (thin line, 1) and after (thick line, 2) 2 $\mu \mathrm{M}$ latrunculin B application. Inset $b$, Before (thin line, 1 ) and after (thick line, 2) $0.3 \mu \mathrm{M}$ CNQX application. Calibration: 3.5 $\mathrm{mV}, 90 \mathrm{msec}$. $C$, Effects of postsynaptically applied latrunculin $\mathrm{B}$ on PPF as measured using whole-cell recording. The bar indicates the duration of drug application to the postsynaptic cell. Latrunculin B $(100 \mu \mathrm{M})$ was applied after $10 \mathrm{~min}$ of baseline recording $(n=7)$. As a control, $0.2 \%$ DMSO internal solution $(n=4)$ was applied. The effect of latrunculin B on PPF was not significantly different from that of DMSO control 50 min after drug application ( $t$ test; $p \gg 0.05)$. Inset, Two example average traces before (thin line, 1) and after (thick line, 2) $100 \mu \mathrm{M}$ latrunculin B application. Calibration: $800 \mathrm{pA}, 120 \mathrm{msec}$. $D$, Effect of bath-applied $4 \mu \mathrm{M}$ latrunculin B on evoked mEPSCs. $a$, An evoked EPSC trace is shown. The arrow indicates when the stimulus was given. The period marked by the gray bar was used for analysis (asterisk indicates mEPSCs). One hundred traces were acquired for each experiment. Calibration: $100 \mathrm{pA}, 500$ msec. A higher concentration of latrunculin B than in Figure 1 was used to speed the onset of the effect. $b$, Latrunculin B significantly reduced mEPSC frequency by $36 \%$. $c$, Cumulative distribution of mEPSC amplitude. Latrunculin B reduced the average mEPSC amplitude by $9 \%$. Amplitude was normalized to $50 \%$ of cumulative percentage in the control condition.
$0.1 \%$ DMSO control $(n=4)$. As an independent control, $0.3 \mu \mathrm{M}$ CNQX, which reduced the slope of fEPSP by $38 \%$ through a postsynaptic action, did not significantly affect PPF $(n=4 ; t$ test; $p \gg 0.05$ at $56-65 \mathrm{~min})$. The fact that latrunculin $\mathrm{B}$ increased PPF but decreased the synaptic response is characteristic of agents that reduce presynaptic release. Consistent with this, we found that intracellular application of $100 \mu \mathrm{M}$ latrunculin B into the postsynaptic cell through a patch electrode (whole-cell recording; see Materials and Methods) produced a small decline of the synaptic response but did not affect PPF (Fig. 3C). These results therefore indicate that latrunculin $\mathrm{B}$ reduces the synaptic response, at least in part, through a presynaptic effect.

As an additional approach to localizing the site of action of bath-applied latrunculin B, we measured the frequency and amplitude of mEPSCs using the whole-cell recording method (Fig. $3 D$ ). $\mathrm{Sr}^{2+}$ (instead of $\mathrm{Ca}^{2+}$ ) was included in the ACSF to produce evoked asynchronous mEPSCs (Miledi, 1966; Goda and Stevens, 1994; Oliet et al., 1996). Thirty minute application of $4 \mu \mathrm{M}$ latrunculin B significantly decreased the frequency of mEPSCs from $11.70 \pm 0.19$ to $7.45 \pm 0.79$, i.e., by $36 \%(n=4$; ANOVA; $p<0.03$ ) (Fig. $3 D b$ ). A change in mini-frequency is usually indicative of a presynaptic site of action. We also found that the average amplitude of mEPSCs was reduced by $9 \%$ from
$6.05 \pm 0.11$ to $5.51 \pm 0.15 \mathrm{pA}$ (Fig. $3 D c$ ). This small decrease was significant (K-S test; $Q \ll 0.01)$. The large reduction of mEPSC frequency and the change in PPF suggest that the primary effect of bath-applied latrunculin B (at early times after application) is presynaptic. The results presented later show more clearly that latrunculin B also affects postsynaptic processes.

\section{LTP induction is inhibited by actin polymerization inhibitors}

To investigate the effect of bath-applied APIs on LTP, we applied APIs for $90 \mathrm{~min}$ before LTP induction. The fEPSP decreased gradually, as shown in Figure $1 A$. After 80 min of application, stimulus strength was increased to bring the fEPSP to its original level, and 10 min later, LTP was induced using a theta-burst protocol (10 bursts of $100 \mathrm{~Hz}$, five pulses every $200 \mathrm{msec}$ ). Washout was done $10 \mathrm{~min}$ after LTP induction. This produced a small recovery of the fEPSP. Figure $4 A$ compares the LTP induced under control conditions $(0.1 \%$ DMSO; average of five experiments) (Fig. 4Aa) with effects of the same theta-burst stimulation done in the presence of either $2 \mu \mathrm{M}$ latrunculin B (average of eight experiments) (Fig. $4 A b$ ) or 5 or $10 \mu \mathrm{M}$ cytochalasin D (average of seven and four experiments, respectively) (Fig. 4Ac). In each case, the response of the LTP pathway is 
Figure 4. Bath-applied latrunculin B and cytochala$\sin$ D inhibit LTP induction. In each experiment, after 80 min of drug application, the stimulus strength was increased to produce the same level of fEPSP slope before drug application. $A$, LTP induction was significantly inhibited by APIs. Arrow indicates the time when the theta-burst stimulus was given. Gray bar indicates the period of the application of drug. $a$, LTP was induced by a theta burst in $0.1 \%$ DMSO $(n=9$; filled rectangles). The magnitudes of average fEPSP of LTP and non-LTP pathways measured 50 min after LTP induction was 139 and $87 \%$ of the baseline, which gave $160 \%$ of potentiation relative to fEPSP of non-LTP path (open rectangles). $b$, latrunculin B $(2 \mu \mathrm{M})$ significantly inhibited LTP $(n=8$; filled circles). The average fEPSPs of LTP and nonLTP path at 50 min after induction were 104 and $92 \%$ of the baseline, which gave $113 \%$ potentiation compared with the fEPSP of non-LTP path (open circles). $c$, Cytochalasin D also inhibited LTP induction. Cytochalasin $\mathrm{D}$ at 5 (rectangles; $n=7$ ) and 10 (triangles; $n=4) \mu \mathrm{M}$ were tested. The average fEPSPs of LTP ( filled) and non-LTP path (open) at $50 \mathrm{~min}$ after induction were 111 and $101 \%$ of the baseline, which gave $110 \%$ relative potentiation in $10 \mu \mathrm{M}$ cytochalasin D experiment. The relative potentiation at $50 \mathrm{~min}$ after LTP induction in $5 \mu \mathrm{M}$ cytochalasin D experiment was $116 \%$. $d$, Cumulative distribution of the ratio of LTP path EPSP over non-LTP path EPSP from individual experiments, measured at 45-50 min after LTP induction. Open circles are for DMSO control experiments $(n=9)$, filled circles are for latrunculin B experiments $(n=5)$, and triangles and rectangles are for $5(n=7)$ and $10(n=4) \mu \mathrm{M}$ cytochalasin D, respectively. The shift of the distribution produced by APIs suggests that LTP induction was reduced significantly $(\mathrm{K}-\mathrm{S}$ test; $Q \ll$ 0.01). $B$, Latrunculin did not strongly affect the temporal pattern of vesicle release during $25 \mathrm{~Hz}$ theta-burst induction protocol. The slope of each fEPSP during theta burst was normalized to that of first fEPSP of the first burst. a, 1st (thin line) and 10th (thick line) burst average traces from control experiment. Calibration: $5 \mathrm{mV}, 200 \mathrm{msec} . b$, The measurement of slope of fEPSPs during theta burst without latrunculin B $(n=4)$. c , Example traces of 1st (thin line) and 10th (thick line) burst under $2 \mu \mathrm{M}$ latrunculin B application. These traces are from the same slice as those shown in $a$. Calibration: $5 \mathrm{mV}, 200 \mathrm{msec} . d$, The measurement of slope of fEPSPs during theta burst with $2 \mu \mathrm{M}$ latrunculin B $(n=4)$.
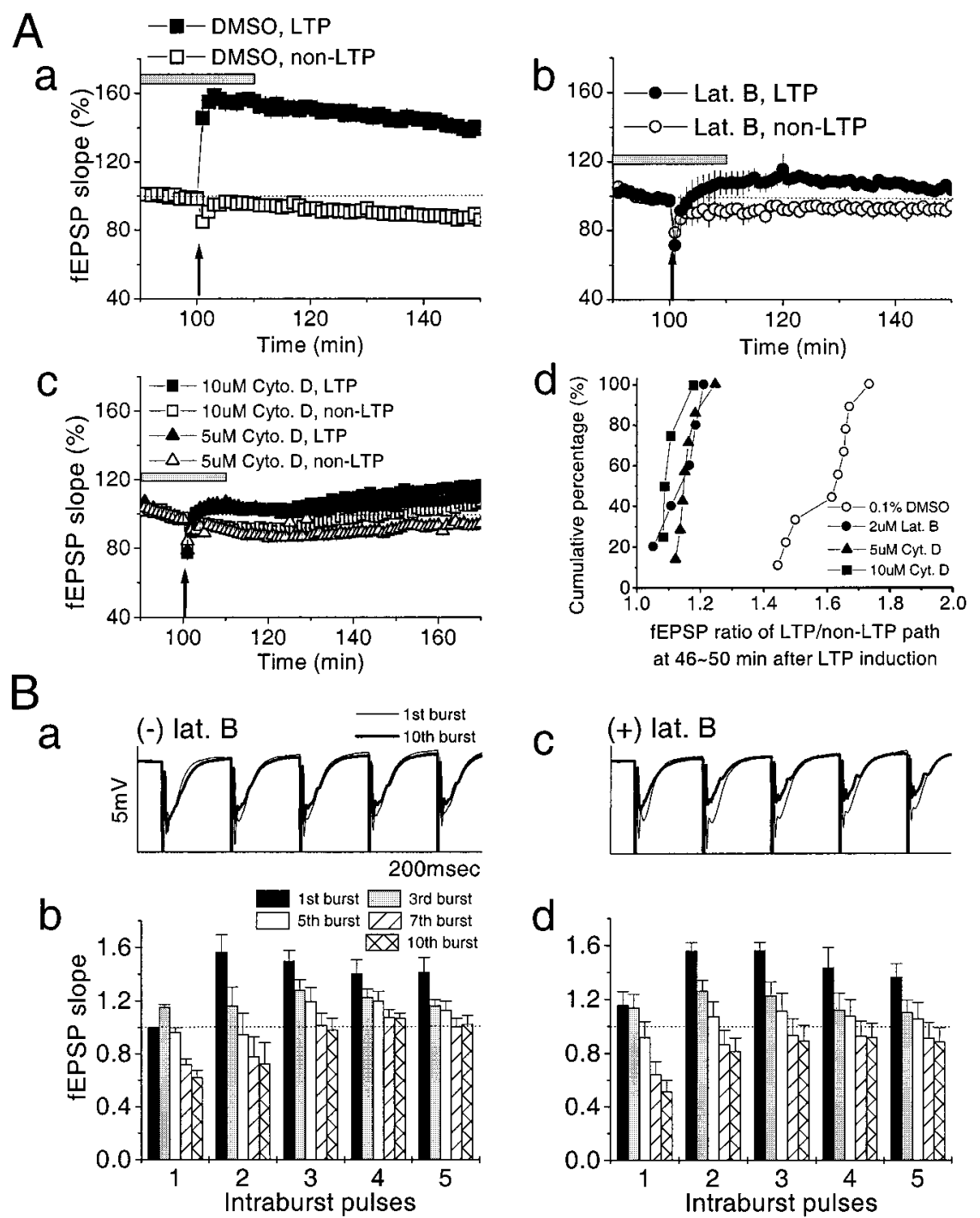

compared with the response from a non-LTP pathway recorded simultaneously. The figure shows a large and obvious reduction in the magnitude of both the initial and maintained LTP. Figure $4 A a$ shows that the non-LTP pathway in DMSO had a small and slow decline $(13 \% / \mathrm{hr})$. This is comparable with that observed in other experiments without DMSO $(11 \% / \mathrm{hr} ; n=6$; data not shown). Because of this small drift, it is most accurate to quantify the magnitude of LTP by the ratio of the size of average fEPSP in the LTP pathway compared with that in the non-LTP pathway. The potentiation of LTP path at 50 min after induction was $160 \%$ in control and $113 \%$ in latrunculin B. Figure $4 \mathrm{Ad}$ shows the cumulative distribution of ratio of LTP over non-LTP path fEPSPs at 46 min after LTP induction.

One possible explanation of the reduction in LTP in API was the interruption of vesicle release or depletion of a releasable vesicle pool during theta-burst stimulation. To explore this possibility, we measured the fEPSPs for each synaptic response during LTP induction. To resolve individual responses, we used a $25 \mathrm{~Hz}$ theta burst rather than a $100 \mathrm{~Hz}$ theta burst. In control experiments, we found that the $25 \mathrm{~Hz}$ protocol could induce LTP and that $2 \mu \mathrm{M}$ latrunculin B inhibited it ( $n=4$; data not shown).
Figure $4 B$ shows the slope of the synaptic response for each stimulus in a burst, for bursts given several times during the induction. It can be seen that latrunculin B did not cause a substantial rundown of transmission during a theta burst. The sum of all fEPSP slopes during the theta burst was decreased only $6 \%$ in the presence of latrunculin B relative to that in its absence (Fig. $4 B c, B d$ ). There was thus no dramatic rundown of transmission during LTP induction that could explain the reduced LTP magnitude.

The results with bath-applied APIs suggest that LTP induction is inhibited because of an action of APIs on postsynaptic actin. To more specifically study postsynaptic effects, latrunculin B was applied postsynaptically through the patch electrode several minutes after the onset of whole-cell recording. In previous experiments (Otmakhov et al., 1997), it was shown that dye applied in this way can take over $30 \mathrm{~min}$ to achieve equilibrium concentration in the distal dendrites. It is not possible to wait for such equilibration before inducing LTP because of problems with LTP "washout." Thus, when LTP was induced 18 min after application of latrunculin B, its concentration in the dendrites must have been considerably lower than that in the internal solution. LTP was induced by the pairing protocol described in Materials and Meth- 


\section{A DMSO internal perfusion}

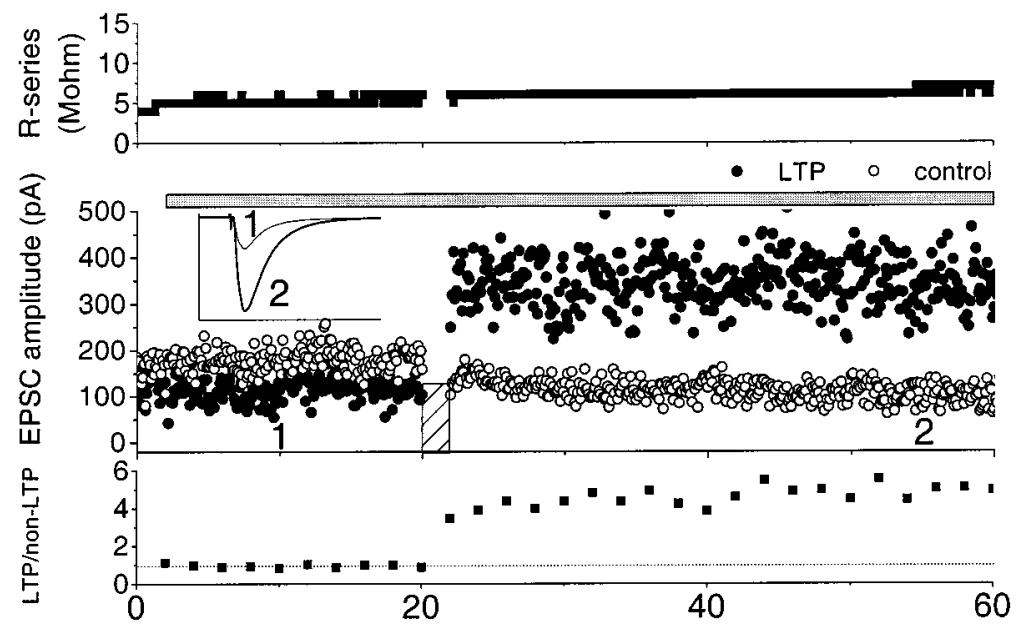

\section{B Latrunculin B + DMSO internal perfusion}

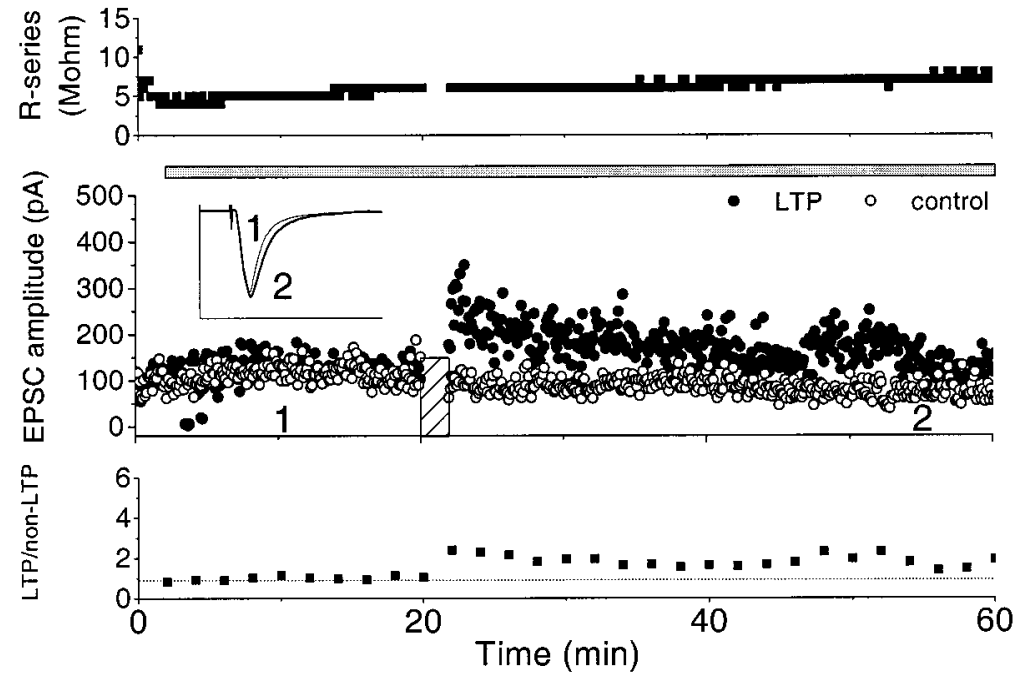

Figure 5. Representative experiments in which effects on LTP induction of postsynaptic DMSO or latrunculin B were measured. Gray bar indicates the period of internal perfusion of latrunculin B or DMSO through perfusion pipette. Hatched box indicates the period of pairing. A, An experiment in which $0.1 \%$ DMSO internal solution was applied for $18 \mathrm{~min}$, and LTP was then induced by pairing. Inset, Average traces for $10 \mathrm{~min}$ before (thin line, 1) and after (thick line, 2) pairing. Calibration: $400 \mathrm{pA}, 60 \mathrm{msec}$. Top panel shows the measurement of series resistance for the recording. Middle panel shows the magnitude of EPSC as a function of time. Bottom panel shows the ratio of amplitude of LTP over non-LTP path synaptic responses. Each point is the average of 20 traces. $B$, An experiment in which $200 \mu \mathrm{M}$ latrunculin B (in $0.1 \%$ DMSO) was applied postsynaptically. Eighteen minutes later, LTP was induced by pairing. Inset, Average trace $10 \mathrm{~min}$ before (thin line, 1) and after (thick line, 2) pairing. Calibration: $250 \mathrm{pA}, 60 \mathrm{msec}$. ods. Figure 5 shows an example of a control experiment $(0.1 \%$ DMSO applied by internal perfusion) and an example in which $200 \mu \mathrm{M}$ latrunculin B in $0.1 \%$ DMSO was perfused. Fig. $6, A$ and $B$, shows summary data for all such experiments $(80-100 \mu \mathrm{M})$, and Figure $6 C$ shows the cumulative distribution of the LTP pathway relative to the non-LTP pathway at $30 \mathrm{~min}$ after LTP induction. Several conclusions can be drawn from these results. First, the magnitude of the initial potentiation $(2 \mathrm{~min}$ after pairing) was reduced by latrunculin B by $42 \%$ ( $t$ test; $p<$ $0.05)$. This reduction occurred at a time when there was little, if any, change in baseline transmission. This indicates an effect on plasticity that cannot be attributed to any generalized reduction in synaptic transmission. Second, within 40-60 min after application of latrunculin $\mathrm{B}$, baseline transmission fell. At $50 \mathrm{~min}$, the reduction was $40 \%$. This is considerably larger than the $17 \%$ reduction in the DMSO control. This indicates that latrunculin B produces a decrease in baseline transmission that develops slowly with time. The third conclusion has to do with the magnitude of LTP measured at $50 \mathrm{~min}$ after induction. Although the potentiation is clearly smaller in latrunculin B than in controls, one might ask whether this is simply because of the smaller initial LTP and a subsequent decay similar to that which occurs in the non-LTP pathway ( $40 \%$ over $50 \mathrm{~min}$ ). If the initial potentiation observed in latrunculin B (207\%) decays at this rate, the expected final level of potentiation is $124 \%$, close to the observed value $(133 \%)$. We conclude that latrunculin $\mathrm{B}$ reduces potentiation by $42 \%$ and that, on a longer time scale, transmission in both LTP and non-LTP pathways decays in a proportional way. Latrunculin B produced little effect on membrane resistance during these experiments ( $<5 \%$ decay; data not shown).

Because interaction of postsynaptic actin filaments with NMDARs has been suggested (Rosenmund and Westbrook, 1993; Wyszynski et al., 1997) and because of the requirements of the NMDAR activation for LTP induction, it was important to test whether postsynaptic application of latrunculin B affected the NMDAR current. The results (Fig. 6D) show that latrunculin B $(80-100 \mu \mathrm{M})$ did not affect the NMDAR component of synaptic transmission isolated by $10 \mu \mathrm{M} \mathrm{CNQX}$ and $1.3 \mathrm{~mm} \mathrm{Mg}^{2+}$.

\section{The actin filament stabilizer phalloidin inhibits basal AMPAR-mediated synaptic transmission and the induction and maintenance of LTP}

It was of interest to determine whether the postsynaptic effects of interfering with actin could be observed using drugs that 
A

Figure 6. Postsynaptically applied latrunculin B reduces pairing-induced LTP without affecting NMDAR-mediated EPSC. Gray bar indicates the period of internal perfusion of latrunculin B or DMSO through perfusion pipette. Arrow indicates the pairing. $A$, In control, $\leq 0.4 \%$ DMSO was applied starting $2 \mathrm{~min}$ after the initiation of whole-cell recording $(n=26)$. Eighteen minutes later, LTP was induced by pairing in one pathway (filled rectangles). The initial average potentiation measured at 2 min after induction was $283 \%$ of the baseline. The average EPSCs of LTP path and non-LTP path (open rectangles) at $30 \mathrm{~min}$ after pairing were 267 and $85 \%$, respectively. $B$, Postsynaptic latrunculin B reduced pairing-induced LTP. Latrunculin B (100 $\mu \mathrm{M} ; n=$ $16 ; 80 \mu \mathrm{M} ; n=2$ ) in DMSO (total, $n=18$ ) was internally applied 2 min after whole-cell recording, and then pairing was applied $18 \mathrm{~min}$ later. The initial average potentiation measured at 2 min after induction was $207 \%$ of the baseline (filled circles). The average EPSCs $(n=18)$ in the LTP path and in the non-LTP path (open circles) at $30 \mathrm{~min}$ after pairing were 156 and $69 \%$ of the baseline, respectively (those values with $100 \mu \mathrm{M}$ latrunculin B were 150 and 68\%; those with $80 \mu \mathrm{M}$ were 194 and 101\%). C, Cumulative distribution of the ratio of EPSC in LTP path over that in non-LTP path from individual experiments, measured 30 min (asterisk in $A$ and $B$ ) after LTP induction. Open circles are for DMSO control experiments $(n=26)$, and filled circles are for latrunculin B experiments $(n=18)$. The shift of distribution by postsynaptic latrunculin B indicates that LTP induction measured at this time is reduced significantly (K-S test; $Q \ll 0.01$ ). $D$, NMDAR-mediated EPSC was not significantly changed by postsynaptic latrunculin $\mathrm{B}$. The bath solution contained $10 \mu \mathrm{M} \mathrm{CNQX}$ and $1.3 \mathrm{~mm} \mathrm{Mg}^{2+}$. Latrunculin B (100 $\mu \mathrm{M} ; n=10 ; 80 \mu \mathrm{M} ; n=5)$ was internally perfused starting $6 \mathrm{~min}$ after whole-cell recording (total, $n=$ 17). The average EPSC $(n=17)$ at $40 \mathrm{~min}$ after latrunculin B application was $96 \%$ (that with $100 \mu \mathrm{M}$ was $97 \%$; that with $80 \mu \mathrm{M}$ was $94 \%$ ). As a control, $\leq 0.2 \%$ DMSO internal solution was perfused $(n=23)$. Inset, Representative average traces of NMDAR EPSC 8 min before (thin line, 1) and after (thick line, 2) $80 \mu \mathrm{M}$ latrunculin B application. Calibration: $60 \mathrm{pA}, 90 \mathrm{msec}$.

B

C
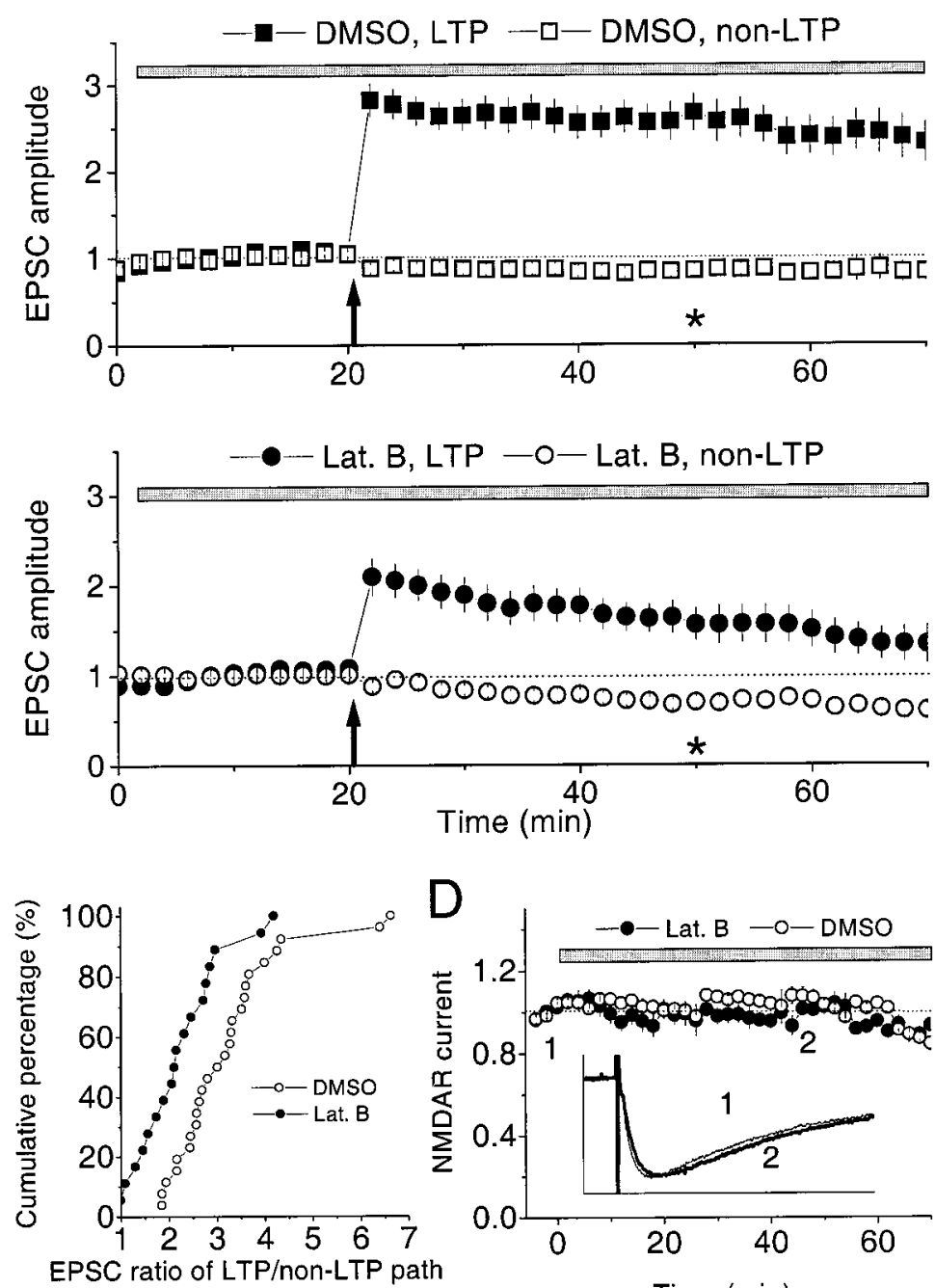

at $30 \mathrm{~min}\left(^{*}\right)$ after LTP induction

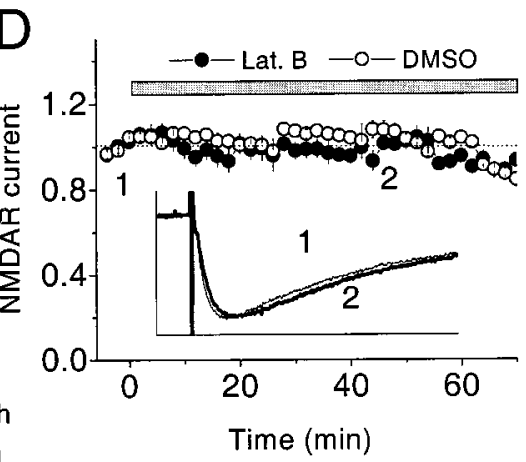

initial potentiation (4 min after pairing) was $243 \%(n=8)$ (Fig. $8 A)$. In phalloidin, the initial potentiation was much smaller $(178 \%)$, i.e., a $45 \%$ drop of potentiation $(n=7 ; t$ test; $p<0.05)$ (Fig. $8 B$ ). Figure $8 C$ shows the cumulative distribution of results. Over the next $50 \mathrm{~min}$, the non-LTP pathway decayed by $54 \%$. This decay was not caused by general deterioration of the cell because the membrane resistance during this period decreased $<10 \%$ (Fig. $8 B$ ). If the LTP pathway decayed at the same rate as the non-LTP path, the potentiation at $50 \mathrm{~min}$ would be $96 \%$, in good agreement with the observed value (99\%). Thus, as with latrunculin B, the effect of phalloidin can be understood as a drop in the initial potentiation, followed by a proportional decay of both non-LTP and LTP pathways.

In the next series of experiments (Fig. 9), we tested whether the maintenance of LTP could be affected by phalloidin. First, LTP was induced, and 2 min later phalloidin was internally applied. Over the next 30 min there was a decay in both the LTP and non-LTP pathway followed by little further decrease (Fig. 9B). During this decay, there was no change in membrane resistance (Fig. 9B). Phalloidin caused a drop in the LTP pathway over time that was somewhat larger than in the non-LTP pathway (Fig. 9C).

Figure $9 D$ shows the distribution of individual experiments. was applied postsynaptically for $18 \mathrm{~min}$, and a pairing stimulus was then given. Under control condition ( $0.1 \%$ DMSO), the 
A

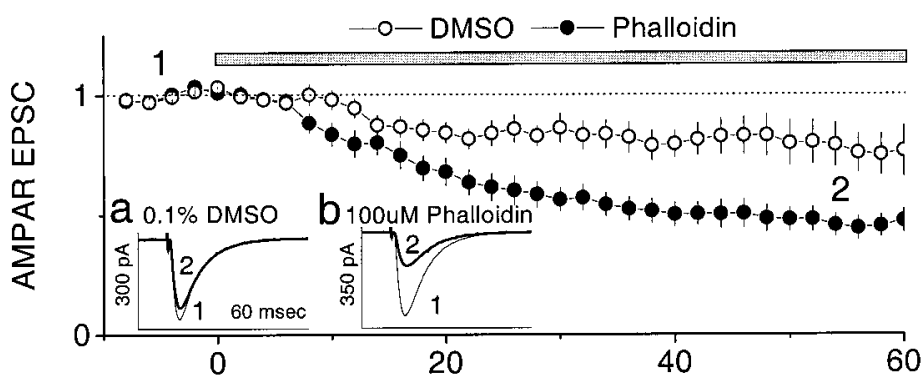

B

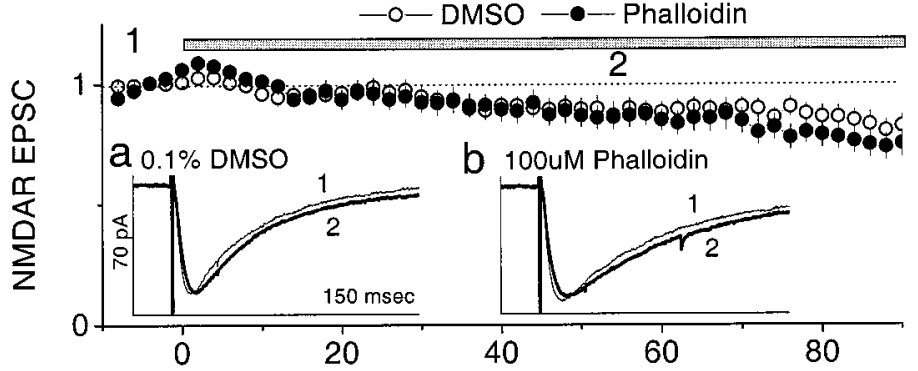

C

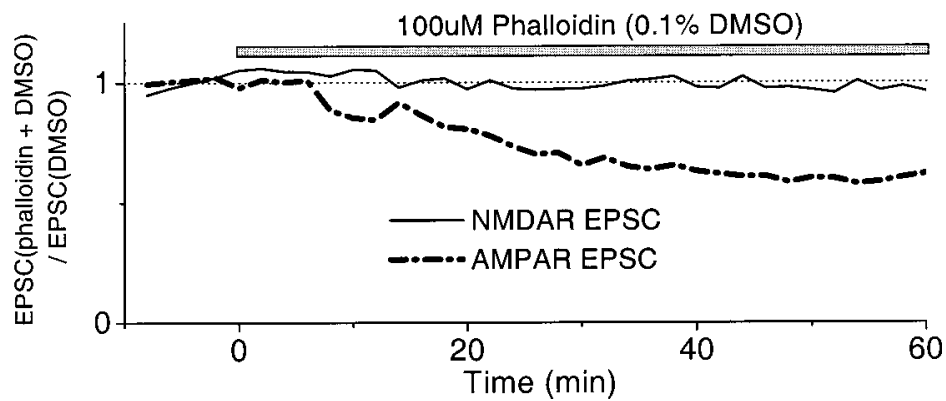

Figure 7. Postsynaptically applied phalloidin reduces the AMPAR-mediated EPSC but does not affect the NMDARmediated EPSC. Gray bar indicates the period of internal perfusion of phalloidin or DMSO. $A$, Effect of $100 \mu \mathrm{M}$ phalloidin (in $0.1 \%$ DMSO; $n=20$ ) on basal AMPAR-mediated synaptic transmission compared with that with $0.1 \%$ DMSO alone $(n=$ $14)$. Results are the average of the number of experiments $(n)$. Drug was applied $10 \mathrm{~min}$ after initiation of whole-cell recording. Holding voltage, $-65 \mathrm{mV}$. Insets $a$ and $b$ show the example average traces for $10 \mathrm{~min}$ before (1) and $50 \mathrm{~min}$ after (2) phalloidin or DMSO alone application, respectively. $B$, Effect of $100 \mu \mathrm{M}$ phalloidin (in $0.1 \%$ DMSO; $n=24$ ) on the NMDARmediated EPSC compared with that with $0.1 \%$ DMSO alone $(n=18)$. Membrane potential was held at -50 to $-65 \mathrm{mV}$ (adjusted in each experiment to give $\sim 70 \mathrm{pA}$ response). Drug was applied $10 \mathrm{~min}$ after initiation of whole-cell recording. Application $(50 \mathrm{~min})$ of phalloidin did not significantly reduce the NMDAR-mediated EPSC amplitude compared with that with DMSO alone ( $t$ test; $p \gg 0.05)$. Insets $a$ and $b$ show the example average traces for $10 \mathrm{~min}$ before (1) and after (2) phalloidin or DMSO alone application, respectively. Calibration: $70 \mathrm{pA}, 150$ msec. $C$, Effect of phalloidin on AMPAR- and NMDARmediated EPSCs. The effect of phalloidin was measured as the ratio of the average EPSC with phalloidin application over that with DMSO alone. Thin trace shows the ratio for the NMDARmediated EPSC measurements shown in B. Thick broken trace shows the ratio for the AMPAR-mediated EPSC measurements shown in $A$.

\section{DISCUSSION}

Because of the high concentration of actin in spines and presynaptic terminals and the close association of actin with the postsynaptic density and ion channels, it has been suspected that actin might play a functional role in synaptic transmission and synaptic plasticity (Fifkova and Morales, 1992; Pavlik and Moshkov, 1992; Edwards, 1995). In this paper, we provide the first functional evidence that actin plays an important role in both presynaptic and postsynaptic processes that contribute to basal synaptic transmission. Perhaps the most surprising finding is that postsynaptic actin is required to maintain AMPAR-mediated synaptic transmission but not NMDAR-mediated synaptic transmission. Our results further show that LTP is reduced by interfering with actin, even at times when basal synaptic transmission is not yet affected.

Because actin is so important as a structural protein, there is the concern that the effects of agents that interfere with actin filaments might be a result of nonspecific effects or of gross changes in cell morphology. However, nonspecific effects cannot account for our data because LTP was reduced at a time when baseline transmission was unaffected (Figs. 6B, 8B). Furthermore, with longer applications of latrunculin $B$ or shorter applications of phalloidin, baseline synaptic transmission was affected, but membrane resistance (Figs. 8B, 9B) and NMDAR-mediated transmission (Figs. $6 D, 7 B$ ) were not. The effects were thus not a result of a nonspecific decline of cell function. It also appears unlikely that the effects were caused by gross structural change.
We examined cell structure in the case of latrunculin B. Although our results cannot exclude small morphological changes, there was certainly no indication of any gross change; spines and dendrites remained clearly identifiable after treatment with latrunculin B (Fig. 2). This is consistent with work from several other laboratories showing that APIs do not strongly affect spine shape on the time scale of a few hours (Allison et al., 1998; Fisher et al., 1998). An additional argument against the involvement of gross structural effects is that latrunculin B, which should depolymerize actin filament, and phalloidin, which should stabilize actin filament and not lead to morphological change, have similar effects on synaptic processes. We conclude that the drugs are not interfering with a static structural process but rather with a dynamic process required for maintaining and potentiating synapses (see below for specific possibilities).

\section{Presynaptic role of actin in basal synaptic transmission}

We have found that bath-applied APIs (cytochalasin D and latrunculin B) reduce synaptic transmission in part by acting at a presynaptic site. Both the AMPAR and NMDAR components of the response were reduced nearly to the same extent at early times after bath application (Figs. $1 A, 3 A$ ), consistent with a presynaptic site of action. Furthermore, bath-applied latrunculin B enhanced paired-pulse facilitation, an enhancement that is typically associated with perturbations that reduce the probability of transmitter release (Creager et al., 1980; Charlton et al., 1982; 
Figure 8. Postsynaptically applied phalloidin reduces the LTP induced by pairing. Gray bar indicates the period of internal perfusion of phalloidin or DMSO, and thick arrow indicates pairing. Error bars indicate SEM. $A$, In controls, $0.1 \%$ DMSO was perfused $2 \mathrm{~min}$ after the initiation of whole-cell recording $(n=8)$, and LTP was induced 18 min later. The initial average potentiation measured $4 \mathrm{~min}$ after induction was $243 \%$ to the baseline (filled rectangles). The average EPSCs in the LTP path and in the non-LTP path (open rectangles) measured at $50 \mathrm{~min}$ after induction were 278 and $104 \%$ to the baseline, respectively. B, Phalloidin $(100 \mu \mathrm{M})$ was internally applied $2 \mathrm{~min}$ after the initiation of whole-cell recording $(n=7)$, and LTP was induced by pairing $18 \mathrm{~min}$ later. The initial average potentiation measured at 4 min after induction was $178 \%$ of the baseline (filled circles). The average EPSCs in the LTP path and in the non-LTP path (open circles) measured at 50 min after Bottom traces show the input resistance and the series resistance as a function of time, respectively. $C$, Cumulative distribution of the ratio of EPSC in the LTP path over that in the non-LTP path at $30 \mathrm{~min}$ (asterisks in $B$ and $C$ ) after LTP induction. The distribution in phalloidin ( filled circles; $n=5$ ) is significantly shifted from that in control DMSO reduction of LTP at 30 min after induction caused by phalloidin (K-S test; $Q \ll 0.01)$. induction were 99 and $46 \%$ of the baseline, respectively. experiment (open circles; $n=8$ ), indicating a significant

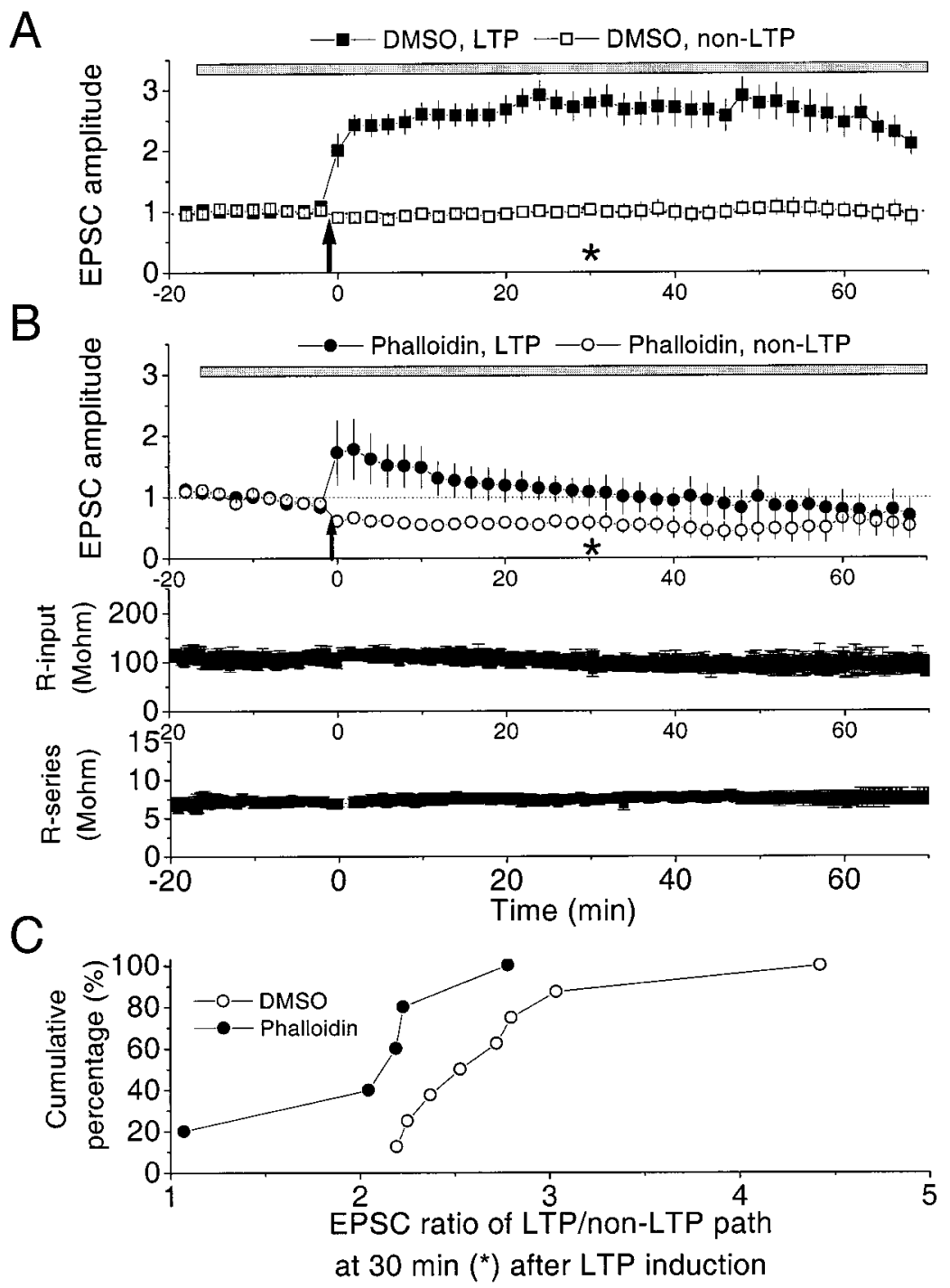

Hess et al., 1987) (Fig. 3B). Direct evidence that latrunculin B does not affect PPF through postsynaptic action was its failure to affect PPF when applied postsynaptically through a patch electrode (Fig. 3C). Similarly, postsynaptically applied phalloidin did not affect PPF ( $n=8$; data not shown). We furthermore found that bath-applied latrunculin $\mathrm{B}$ produced a large reduction in the frequency of miniature synaptic responses but produced, at most, a small reduction in the amplitude of miniature synaptic responses (30-40 min after API application) (Fig. 3D). Together, these results indicate that bath-applied latrunculin B can act at a presynaptic site to reduce basal synaptic transmission.

One possible mechanism of these effects relates to presynaptic $\mathrm{Ca}^{2+}$ channels. It has been shown that actin depolymerization can attenuate $\mathrm{Ca}^{2+}$ entry through calcium channels (Furukawa et al., 1995) and speed the rundown of calcium channels (Johnson and Byerly, 1993). Such effects could account for the decreased transmission and enhancement of PPF that we have observed.

\section{Postsynaptic role of actin in basal synaptic} transmission and LTP

Our results with postsynaptically applied actin function inhibitors indicate that there is also a postsynaptic role for actin in maintaining basal synaptic transmission. Postsynaptically applied phal- loidin produced a large $(\sim 40 \%)$ reduction in synaptic transmission after 30 min (Figs. $7 A, 8 B, 9 B$ ). Latrunculin $\mathrm{B}$ also reduced transmission, but the effect developed more slowly. These results suggest that basal AMPAR-mediated synaptic transmission is dependent on an actin-mediated process. In contrast, neither postsynaptic application of latrunculin B nor phalloidin affected NMDAR-mediated transmission. Our results with phalloidin are consistent with previous work showing that phalloidin does not inhibit the NMDAR current and in fact prevents its usedependent rundown (Rosenmund and Westbrook, 1993).

Our result showing that basal AMPAR-mediated synaptic transmission and LTP can be selectively inhibited by interfering with actin function has intriguing similarities to recent work showing that postsynaptic application of a peptide that interferes with the interaction of $\mathrm{N}$-ethylmaleimide-sensitive factor (NSF) and glutamate receptor subtype 2 produces a decline in basal synaptic transmission within $\sim 30 \mathrm{~min}$, comparable with the time course we find with actin inhibitors (Nishimune et al., 1998; Song et al., 1998). Furthermore, just as with the NSF inhibitory peptide, the actin inhibitors induce a decline in transmission, which is not complete and appears to plateau at a level of $\sim 50 \%$ (Figs. $7 A, 8 B$, $9 B$ ). One possible explanation is that there are two components of 
A

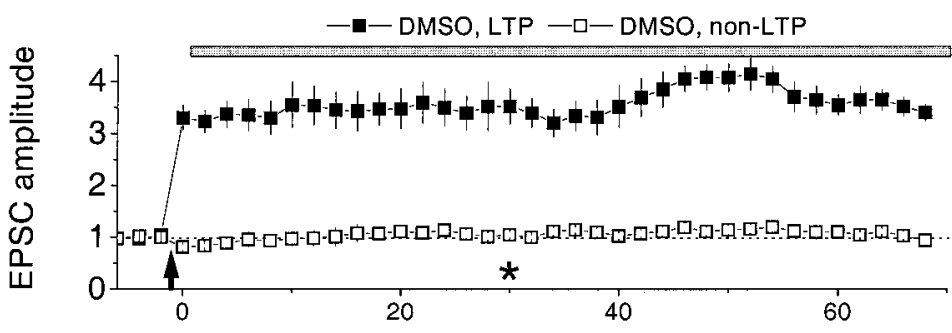

B
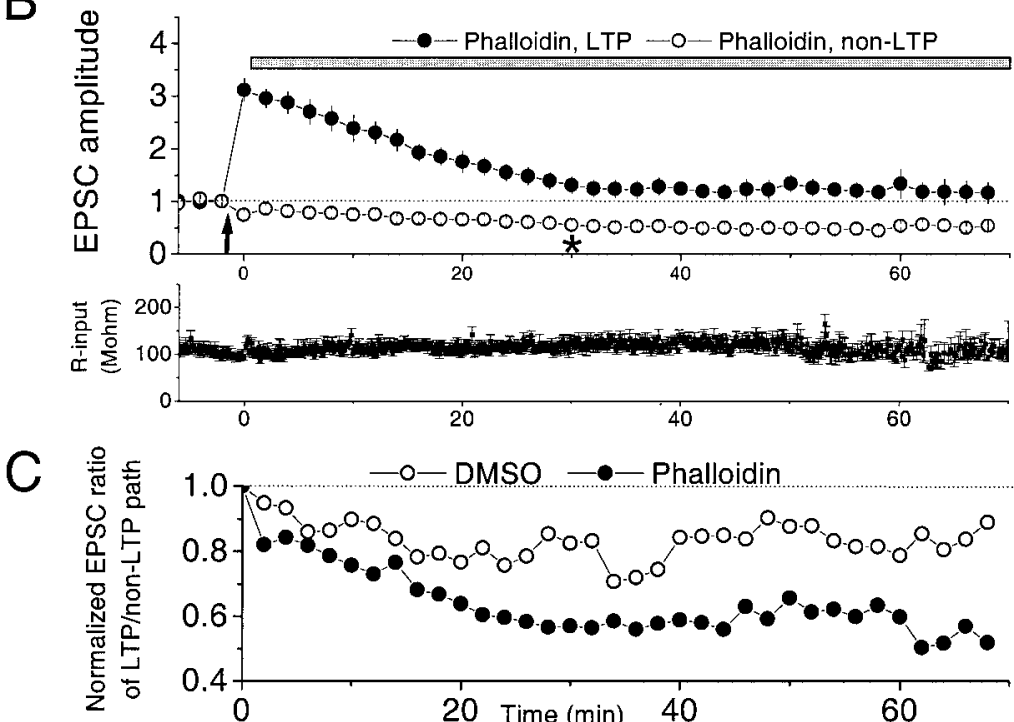

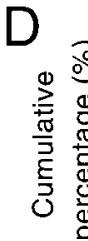

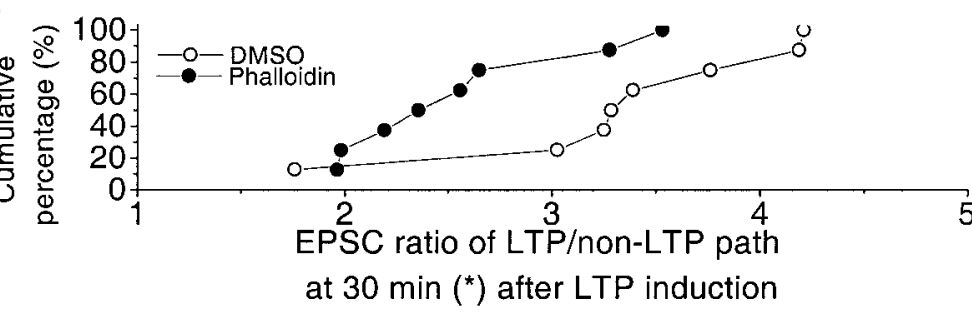

Figure 9. Postsynaptically applied phalloidin reduces the maintenance of pairing-induced LTP. Gray bar indicates the period of internal perfusion of phalloidin or DMSO, and thick arrow indicates pairing. Error bars indicate SEM. $A$, In control $(n=8), 0.1 \%$ DMSO was applied 2 min after LTP induction by pairing ( filled rectangles). The average EPSCs in the LTP and in the non-LTP pathway (open rectangles) measured 2 min after pairing were 330 and $81 \%$ of the baseline. The average EPSCs in the LTP path and in the non-LTP path measured at $50 \mathrm{~min}$ after induction were 407 and $114 \%$ of the baseline, which gave $357 \%$ of relative potentiation. $B$, Phalloidin $(100 \mu \mathrm{M})$ was perfused $2 \mathrm{~min}$ after pairing $(n=9)$. The average EPSCs in the LTP path (filled circles) and in the non-LTP path (open circles) measured at 2 min after induction were 311 and $74 \%$ of the baseline. The average EPSCs in the LTP path and in the non-LTP path measured at 50 min after induction were 134 and $49 \%$ of the baseline, which gave $273 \%$ of relative potentiation. Therefore, phalloidin application reduced the potentiation of LTP by $33 \%$ at $50 \mathrm{~min}$ after induction. Bottom panel shows the input resistance as a function of time. $C$, Ratio of average EPSC in the LTP path over that in the non-LTP path as a function of time. The ratio was normalized to the ratio at 2 min after induction. The larger decay in the ratio was produced by phalloidin ( filled circles; $n=9$; from $B$ ) compared with that with DMSO control (open circles; $n=8$; from $A$ ), which indicates a more selective effect of phalloidin on LTP maintenance. Cumulative distribution of the ratio of EPSC in the LTP path to that in the non-LTP path measured at $30 \mathrm{~min}$ after LTP induction (asterisks in $A$ and $B$ ). The ratio from phalloidin experiment ( filled circles; $n=8$ ) was significantly shifted from that from control DMSO experiment (open circles; $n=8$ ), indicating a significant reduction in LTP at 30 min after induction by postsynaptic phalloidin (K-S test; $Q \ll 0.01$ ).
AMPAR-mediated synaptic transmission: one that is sensitive to NSF inhibitory peptide and actin inhibitors, and one that is not.

One possible mechanism of the effect of NSF inhibitory peptide could be attributable to a change in clustering of AMPARs (Xie et al., 1997; Song et al., 1998), and actin could also be involved in this process. Some evidence for a role of actin in clustering has been provided by Allison et al. (1998), who found that prolonged (24 hr) treatment with $5 \mu \mathrm{M}$ latrunculin A reduced the number of spines and AMPAR clusters. However, it remains unclear whether declustering occurs on the much faster time scale of our experiments. Another possibility of the role of NSF (Song et al., 1998) and actin is the delivery of AMPARs to the synaptic membrane. There is increasing evidence that AMPARs are undergoing a rapid basal turnover. Specifically, it has been shown that interfering with NSF leads to a rapid decrease in basal synaptic transmission; conversely, enhancing the postsynaptic exocytotic process by adding exogenous soluble $N$-ethylmaleimidesensitive factor attached protein, another vesicle fusion protein that may deliver new vesicles to the synapse, enhances AMPARmediated synaptic transmission within 30 min (Lledo et al., 1998). Interfering with vesicle fusion process blocks LTP induction, perhaps because new AMPARs are required. If rapid turnover of AMPAR is occurring by a vesicle-mediated fusion process, in- hibiting actin might interfere with vesicle delivery (Linstedt and Kelly, 1987; Bernstein and Bamburg, 1989; Vitale et al., 1995) and thereby reduce both basal synaptic transmission and LTP. It should be emphasized, however, that relatively little is known about any of these processes. It is not yet clear whether the NSF-exocytosis process occurs near synapses or whether it actually delivers AMPARs to the plasma membrane. Similarly, many possible explanations of the effect of actin inhibitors remain. One actin-dependent process that has been identified is the submicrometer movement of spines (Fisher et al., 1998). These movements are rapidly blocked by APIs and could be related to the physiological effects we have observed.

Interfering with postsynaptic actin also reduces the magnitude of LTP. Similar results were obtained with both latrunculin B and phalloidin (Figs. $4 A b, 4 A c, 6 B, 8 B$ ). Importantly, these effects on LTP occurred at a time after drug application when there was no appreciable effect on basal synaptic transmission. This indicates that LTP induction is more sensitive to actin inhibitors than basal synaptic transmission. We furthermore tested whether phalloidin, when applied after induction, would interfere with LTP maintenance and whether there was any difference in the way it affected the LTP and control pathway. Our results suggest that the LTP pathway is more strongly affected (Fig. 9B,C). In contrast, when 
phalloidin was applied before LTP induction, the two pathways were reduced proportionally.

There are several explanations for why LTP may be dependent on actin filament. First, as suggested by Edwards (1995), actin filament may be required to split the active zone into multiple independent ones. Second, actin filaments in spines may undergo a reversible gel-sol-gel transition during LTP induction, as suggested by Fifkova and Morales (1992). Block of this transition by API or phalloidin may prevent other changes required for LTP expression. Third, actin might be required to cluster extrasynaptic AMPARs in the synapse (Xie et al., 1997). Fourth, actin filaments may be required for a vesicle fusion process that delivers more AMPAR to the synapse during LTP (see above). Because there are specific agents that interfere with membrane fusion processes, it should now be possible to test whether the fusion-dependent processes and actin-dependent processes are along the same pathway controlling AMPAR-mediated transmission.

\section{REFERENCES}

Allison DW, Gelfand VI, Spector I, Craig AM (1998) Role of actin in anchoring postsynaptic receptors in cultured hippocampal neurons: differential attachment of NMDA versus AMPA receptors. J Neurosci 18:2423-2436.

Bernstein BW, Bamburg JR (1989) Cycling of actin assembly in synaptosomes and neurotransmitter release. Neuron 3:257-265.

Bortolotto ZA, Collingridge GL (1998) Involvement of calcium/ calmodulin-dependent protein kinases in the setting of a molecular switch involved in hippocampal LTP. Neuropharmacology 37:535-544.

Calakos N, Scheller RH (1996) Synaptic vesicle biogenesis, docking, and fusion: a molecular description. Physiol Rev 76:1-29.

Charlton MP, Smith SJ, Zucker RS (1982) Role of presynaptic calcium ions and channels in synaptic facilitation and depression at the squid giant synapse. J Physiol (Lond) 323:173-193.

Cohen GA, Doze VA, Madison DV (1992) Opioid inhibition of GABA release from presynaptic terminals of rat hippocampal interneurons. Neuron 9:325-335.

Cohen RS, Chung SK, Pfaff DW (1985) Immunocytochemical localization of actin in dendritic spines of the cerebral cortex using colloidal gold as a probe. Cell Mol Neurobiol 5:271-284.

Collingridge GL, Kehl SJ, McLennan H (1983) Excitatory amino acids in synaptic transmission in the Schaffer collateral-commissural pathway of the rat hippocampus. J Physiol (Lond) 334:33-46.

Cooper JA (1987) Effects of cytochalasin and phalloidin on actin. J Cell Biol 105:1473-1478.

Creager R, Dunwiddie T, Lynch G (1980) Paired-pulse and frequency facilitation in the CA1 region of the in vitro rat hippocampus. J Physiol (Lond) 299:409-424.

Cumming R, Burgoyne RD (1983) Compartmentalization of neuronal cytoskeletal proteins. Biosci Rep 3:997-1006.

Drenckhahn D, Kaiser H-W (1983) Evidence for the concentration of F-actin and myosin in synapses and in the plasmalemmal zone of axons. Eur J Cell Biol 31:235-240.

Edwards FA (1995) Anatomy and electrophysiology of fast central synapses lead to a structural model for long-term potentiation. Physiol Rev 75:759-787.

Fifkova E (1985) Actin in the nervous system. Brain Res Rev 9:187-215.

Fifkova E, Delay RJ (1982) Cytoplasmic actin in neuronal processes as a possible mediator of synaptic plasticity. J Cell Biol 95:345-350.

Fifkova E, Morales M (1992) Actin matrix of dendritic spines, synaptic plasticity, and long-term potentiation. Int Rev Cytol 139:267-307.

Fischer M, Kaech S, Knutti D, Matus A (1998) Rapid actin-based plasticity in dendritic spines. Neuron 20:847-854.

Furukawa K, Smith-Swintosky VL, Mattson MP (1995) Evidence that actin depolymerization protects hippocampal neurons against excitotoxicity by stabilizing $\left[\mathrm{Ca}^{2+}\right]_{\mathrm{i}}$. Exp Neurol 133:153-163.

Geinisman Y, deToledo-Morrell L, Morrell F (1991) Induction of longterm potentiation is associated with an increase in the number of axospinous synapses with segmented postsynaptic densities. Brain Res 566:77-88.
Goda Y, Stevens CF (1994) Two components of transmitter release at a central synapse. Proc Natl Acad Sci USA 91:12942-12946.

Greengard P, Valtorta F, Czernik AJ, Benfenati F (1993) Synaptic vesicle phosphoproteins and regulation of synaptic function. Science 259:780-785.

Gulley RL, Reese TS (1981) Cytoskeletal organization at the postsynaptic complex. J Cell Biol 91:2998-2302.

Hess G, Kuhnt U, Voronin LL (1987) Quantal analysis of paired-pulse facilitation in guinea pig hippocampal slices. Neurosci Lett 77:187-192.

Johnson BD, Byerly L (1993) A cytoskeletal mechanism for $\mathrm{Ca}^{2+}$ channel metabolic dependence and inactivation by intracellular $\mathrm{Ca}^{2+}$. Neuron 10:797-804.

Kaech S, Fischer M, Doll T, Matus A (1997) Isoform specificity in the relationship of actin to dendritic spines. J Neurosci 17:9565-9572.

Kelly PT, Cotman CW (1978) Synaptic proteins. J Cell Biol 79:173-183.

Landis DM (1988) Membrane and cytoplasmic structure at synaptic junctions in the mammalian central nervous system. J Electron Microsc Tech 10:129-151.

Linstedt AD, Kelly RB (1987) Overcoming barriers to exocytosis. Trends Neurosci 10:446-448.

Lledo P-M, Zhang X, Sudof TC, Malenka RC, Nicoll RA (1998) Postsynaptic membrane fusion and long-term potentiation. Science 279:399-403.

Markham JA, Fifkova E (1986) Actin filament organization within dendrite and dendritic spines during development. Dev Brain Res 27:263-269.

Matus A, Ackermann M, Pehling G, Byers HR, Fujiwara K (1982) High actin concentration in brain dendritic spines and postsynaptic densities. Proc Natl Acad Sci USA 79:7590-7594.

Miledi R (1966) Strontium as a substitute for calcium in the process of transmitter release at the neuromuscular junction. Nature 212:1233-1234.

Nishimune A, Isaac JTR, Molnar E, Noel J, Nash SR, Tagaya M, Collingridge GL, Nakanishi S, Henley JM (1998) NSF binding to GluR2 regulates synaptic transmission. Neuron 21:87-97.

Oliet SHR, Malenka RC, Nicoll RA (1996) Bi-directional control of quantal size by synaptic activity in the hippocampus. Science 271:1294-1297.

Otmakhov N, Griffith LC, Lisman JE (1997) Postsynaptic inhibitors of calcium/calmodulin-dependent protein kinase type II block induction but not maintenance of pairing-induced long-term potentiation. J Neurosci 17:5357-5365.

Paoletti P, Ascher P (1994) Mechanosensitivity of NMDA receptors in cultured mouse central neurons. Neuron 13:645-655.

Pavlik LL, Moshkov DA (1992) Actin in synaptic cytoskeleton during long-term potentiation in hippocampal slice. Acta Histochem Suppl 41:257-264.

Rosenmund C, Westbrook GL (1993) Calcium-induced actin depolymerization reduced NMDA channel activity. Neuron 10:805-814.

Song I, Kamboj S, Xia J, Dong H, Liao D, Huganir RL (1998) Interaction of the $N$-ethylmaleimide-sensitive factor with AMPA receptors. Neuron 21:393-400.

Spector I, Shochet NR, Kashman Y, Groweiss A (1983) Latrunculins: novel marine toxins that disrupt microfilament organization in cultured cell. Science 219:493-495.

Spector I, Shochet NR, Blasberger D, Kashman Y (1989) Latrunculins: novel marine macrolides that disrupt microfilament organization and affect cell growth. I. Comparison with cytochalasin D. Cell Motil Cytoskeleton 13:127-144.

Sudhof TC (1995) The synaptic vesicle cycle: a cascade of proteinprotein interactions. Nature 375:645-653.

Vitale ML, Seward EP, Trifaro J-M (1995) Chormaffin cell cortical actin network dynamics control the size of the release-ready vesicle pool and the initial rate of exocytosis. Neuron 14:353-363.

Wigstrom H, Gustafsson B (1986) Postsynaptic control of hippocampal long-term potentiation. J Physiol (Paris) 81:228-236.

Wyszynski M, Lin J, Rao A, Beggs AH, Craig AM, Sheng M (1997) Competitive binding of $\alpha$-actinin and calmodulin to the NMDA receptor. Nature 385:439-442.

Xie X, Liaw J-S, Baudry M, Berger TW (1997) Novel expression mechanism for synaptic potentiation: alignment of presynaptic release site and postsynaptic receptor. Proc Natl Acad Sci USA 94:6983-6988. 